Conclusion: Our small study indicates that clinical and morphological features of renal involvement, as well as renal survival are similar in ANCA-negative and ANCA-positive patients and don't depend on the type of ANCA.

References:

[1] Berden AE, Ferrario F, Hagen EC, et al. Histopathologic Classification of ANCA-Associated Glomerulonephritis. J Am Soc Nephrol. 2010;21(10):1628-1636.

[2] Brix RB, Noriega $M$, Tennstedt $P$, et al. Development and validation of a renal risk score in ANCA-associated glomerulonephritis. Kidney Int. 2018;94(6):1177-1188.

Disclosure of Interests: : Nikolai Bulanov Grant/research support from: This work was supported by the 5-100 Project, Sechenov University, Moscow, Ekaterina Stolyarevich: None declared, Anastasiia Zykova: None declared, Elizaveta Safonova: None declared, Elena Shchegoleva: None declared, Ekaterina Kuznezova: None declared, Mayra Bulanova: None declared, Pavel Novikov Grant/research support from: This work was supported by the 5-100 Project, Sechenov University, Moscow, Sergey Moiseev Grant/research support from: This work was supported by the 5-100 Project, Sechenov University, Moscow

DOI: 10.1136/annrheumdis-2020-eular.6262

\section{AB0498 CHARACTERISTICS AND OUTCOME OF CORONARY ARTERY LESIONS DUE TO POLYARTERITIS NODOSA: ANALYSIS OF A SINGLE CENTER COHORT IN CHINA}

C. Lai ${ }^{1}$, L. Zhao ${ }^{2}$, J. Zhou ${ }^{3}$, D. Xu ${ }^{3}$, X. Tian ${ }^{3}$, X. Zeng ${ }^{3}$, F. Zhang ${ }^{3} .{ }^{1}$ Peking Union Medical College Hospital, Beijing, China; ${ }^{2}$ The Second Hospital of Dalian Medical University, Liaoning, China; ${ }^{3}$ Peking Union Medical College Hospital, Beijing, China

Background: Polyarteritis nodosa (PAN) is a systemic necrotizing vasculitis that mainly affects medium-sized muscular arteries. The coronary artery could be affected. Some severe cases can lead to spontaneous coronary artery dissection (SCAD) and about $0.02 \%$ will die abruptly. Early diagnosis will improve prognosis, but relative studies are all case reports so far.

Objectives: To investigate the clinical characteristics, risk factors and outcome of patients with polyarteritis nodosa (PAN) complicated with coronary artery lesions in China.

Methods: Data of 158 patients with PAN who were admitted to Peking Union Medical College Hospital from September 1986 to September 2019 were retrospective collected. Data were analyzed and compared according to with and without coronary artery lesions due to PAN.

Results: 17 (10.8\%) patients with PAN had the coronary artery lesions due to PAN. The age at coronary artery lesion was $36.9 \pm 10.3$ years. $12(70.6 \%)$ patients were male. There are not statistical differences between two groups in common risk factors of coronary arterial atherosclerosis including smoking, hypertension, diabetes mellitus and hyperlipidemia. Most of them are multi-vessel lesions ( 8 cases are triple-vessel lesions and 3 cases are bi-vessel lesions). Type of coronary artery affected is shown mainly in stenosis (13 cases). Myocardial infarction are shown in 8 cases $(47.1 \%)$. Compared to patients without coronary artery lesions, patients with coronary artery lesions had less nervous system involvement (17.6\% vs.46.8\%) and elevated number of leukocyte (17.6\% vs.56\%). Besides, patients with coronary artery affected exhibit more cranial and carotid artery involvement(29.4\% vs. $5.0 \%)$, renal artery involvement $(41.2 \%$ vs. $17.0 \%)$, coeliac artery involvement (58.8\% vs. $27.0 \%)$, new onset hypertension ( $47.1 \%$ vs. $14.5 \%$ ), renal infarction $(27.3 \%$ vs.5.4\%, ) and higher proportion of 2009 Five-factor score (FFS) $\geq 2$ $(62.5 \%$ vs. $15.6 \%)$. All patients with coronary artery lesions received at least moderate dose of prednisone and CTX except one refused medication. 3 cases underwent interventional therapy. Stent placement was performed on 2 of them, and in-stent restenosis was appeared in a patient one year later. 2 cases died,one for vascular rupture after coronary aneurysmsutrue plus coronary artery bypass grafting, another for myocardial infarction after stopping immunosuppressant therapy himself.Survival analysis showed patients with digital $\mathrm{g}$ angrene had poor prognosis though no significant difference $(p=0.055)$

Conclusion: PAN with coronary artery lessions are not uncommon. These patients exhibit young age, more proportion of multi-vessel of coronary artery involvement, more combined involvments of other organ arteries and more severe disease.

\section{References:}

[1] Munguti CM, Ndunda PM, Muutu TM. Sudden Death From Spontaneous Coronary Artery Dissection Due to Polyarteritis Nodosa. Cureus, 2017;9 (10), e1737

[2] Kritta nawong C, Kumar A, Johnson KW, et al.Conditions and Factors Associated With Spontaneous Coronary Artery Dissection (From a National Population-Based Cohort Study).Am J Cardiol, 2019; 123 (2): 249-253

[3] Hwang J, Yang JH, Kim DK, Cha HS.Polyarteritis Nodosa Involving Rena and Coronary Arteries. J Am Coll Cardiol. 2012;59(7):e13

Disclosure of Interests: : Chinchih Lai: None declared, Lin Zhao: None declared, Jiaxin Zhou: None declared, Dong Xu: None declared, Xinping Tian: None declared, Xiaofeng Zeng Consultant of: MSD Pharmaceuticals, Fengchun Zhang: None declared

DOI: 10.1136/annrheumdis-2020-eular.4343

\section{AB0499 $\quad$ ABNORMALITY OF PERIPHERAL LYMPHOCYTE SUBSETS IN BECHET'S DISEASE AND EFFECTS OF NEW IMMUNOREGULATORY COMBINATION THERAPIES ON THESE CELLS}

Q. Y. Su ${ }^{1}$, S. X. Zhang ${ }^{2}$, L. M. Hao ${ }^{2}$, J.Y. Yang ${ }^{1}$, J. Bai ${ }^{1}$, S. J. Guo ${ }^{1}$, J. Luo ${ }^{2}$, G. Y. Liu' ${ }^{2}, \mathrm{C} . \mathrm{Gao}^{3}, \underline{\mathrm{X} . \mathrm{LI}^{2}} .{ }^{1}$ Shanxi Medical University, Taiyuan, China; ${ }^{2}$ the Second Hospital of Shanxi Medical University, Taiyuan, China; ${ }^{3}$ Brigham and Women's Hospital, Harvard Medical School, Boston, United States of America

Background: Bechet's disease (BD) is a chronic multisystemic vasculitis Although its exact etiopathology is unknown, both autoimmune imbalances associated with genetic and abnormal immune response of effector lymphocytes promoted by infectious factors are suggested ${ }^{1}$. The increase of effector $T$ cells (Teffs) and the decrease of regulatory $T$ cells (Tregs) are possibly the involving factors in the pathogenesis of $\mathrm{BD}^{2}$. Importantly, we have developed new immunoregulatory combination therapies trying to restore the reduction of Tregs in rheumatic patients.

Objectives: To examine abnormal levels of lymphocyte subsets in BD patients at a relatively large-sample size and to investigate whether the immunoregulatory combination therapies have therapeutic efficacy in BD.

Methods: Total 384 BD patients and 206 healthy controls (HCs) were enrolled in this cross-sectional study. Proportions and absolute numbers of peripheral T, $\mathrm{B}, \mathrm{NK}, \mathrm{CD}^{+} \mathrm{T}, \mathrm{CD}^{+} \mathrm{T}, \mathrm{Th} 1, \mathrm{Th} 2, \mathrm{Th} 17$ and Treg subsets were analyzed by flow cytometry (FCM) for all participants. Among these patients, 183 cases of BD patients were treated with immunoregulatory combination drugs (IMiDs) such as low-dose interleukin-2, rapamycin, metformin, retinoic acid and coenzyme Q10. The levels of peripheral lymphocyte subsets were measured before and after the treatment. Compared-T test was used to compare continuous measures and to assess effect of these drugs.

Results: Compared to HCs, the absolute numbers of various Teffs such as T, $\mathrm{B}, \mathrm{CD} 4^{+} \mathrm{T}, \mathrm{CD} 8^{+} \mathrm{T}$, Th1 and Th17 cells were significantly increased in BD group $(P<0.01)$, while the level of Tregs in patients with BD was severely decreased $(P<0.05)$, resulting in increased ratios (imbalance) of Th1/Tregs, Th2/Tregs and Th17/Tregs $(P<0.05)$ (Figure 1). After the IMiDs treatment, the levels of NK, $\mathrm{CD}^{+} \mathrm{T}, \mathrm{CD}^{+} \mathrm{T}$, Th1, Th17 cells as well as Tregs were significantly increased $(P<0.05)$. But the increased Tregs was much more dramatical than those of Teffs, resulting in a decrease in ratios of Teffs/Tregs such as Th2/Tregs $(P<$ 0.001) (Figure 2).

Conclusion: Impaired balance of pro- and anti-inflammatory immune cells, especially insufficiency of Tregs, might be a cornerstone of the pathogenesis of BD. Immunoregulatory combination therapies could promote the proliferation and functional recovery of Tregs in patients with BD and might help to alleviate disease activity.

References:

[1] Yazici H, Seyahi E, Hatemi G, et al. Behcet syndrome: a contemporary view. Nat Rev Rheumatol 2018;14(2):107-19. doi: 10.1038/nrrheum.2017.208 [published Online First: 2018/01/04]

[2] Rosenzwajg M, Lorenzon R, Cacoub P, et al. Immunological and clinical effects of low-dose interleukin-2 across 11 autoimmune diseases in a single, open clinical trial. Ann Rheum Dis 2019;78(2):209-17. doi: 10.1136/annrheumdis-2018-214229 [published Online First: 2018/11/26] 